\title{
Paper A Survey on Data Perception in Cognitive Internet of Things
}

\author{
Lokesh B. Bhajantri ${ }^{1}$ and Prashant Baluragi ${ }^{2}$ \\ ${ }^{1}$ Department of Information Science and Engineering, Basaveshwar Engineering College, Bagalkot, India \\ ${ }^{2}$ Department of Master of Computer Application, KLE Institute of Technology, Hubli, India
}

\begin{abstract}
A Cognitive Internet of Things (CIoT) is a brand of Internet of Things (IoT) with cognitive and agreeable mechanisms, which are incorporated to advance performance and accomplish insights into real world environments. CIoT can perceive present system's conditions, analyze the apparent information, make smart choices, and increase the network performance. In this survey paper, we present classifications of data perception techniques used in CIoT. This paper also compares the data perception works against energy consumption, network life-time, resource allocation, and throughput, as well as quality of data and delay. In addition, simulation tools for IoT and their performance are discussed. Finally, we provide the model of cognitive agent-based data perception in CIoT for future research and development, which ensures the network performance in terms of reliability, energy efficient, accuracy, scalable, fault tolerant, and quality of data.
\end{abstract}

Keywords-Internet of Things, Cognitive IT, data perception, cognitive agents.

\section{Introduction}

Minerva et al. in [1] described that Internet of Things (IoT) is a novel paradigm which enables many relative small devices like sensors/actuators, to acquire and transfer information to distributed frameworks without or with negligible human intervention. The advancement of IoT has brought an awesome impact to lot of applications in then allows to use technologies like social insurance, transportation, assembling, as discussed by Gubbi et al. and Luong et al. in [2], [3]. The IoT associates every one of the things in the physical world to the Internet, and cloud computing, deep learning, cognitive computing. The communication utilizing IoT for different applications is given in Fig. 1. This figure comprises with three layers for IoT communications is as follows:

- Mobile-to-mobile (M2M) device. A "thing" (in IoT) is an object equipped with sensors or/and actuators. Sensors gather data which next are transferred over a network. Actuators allow things to act (for example, to switch on or off the light. This concept includes the sensors in various domains like medical

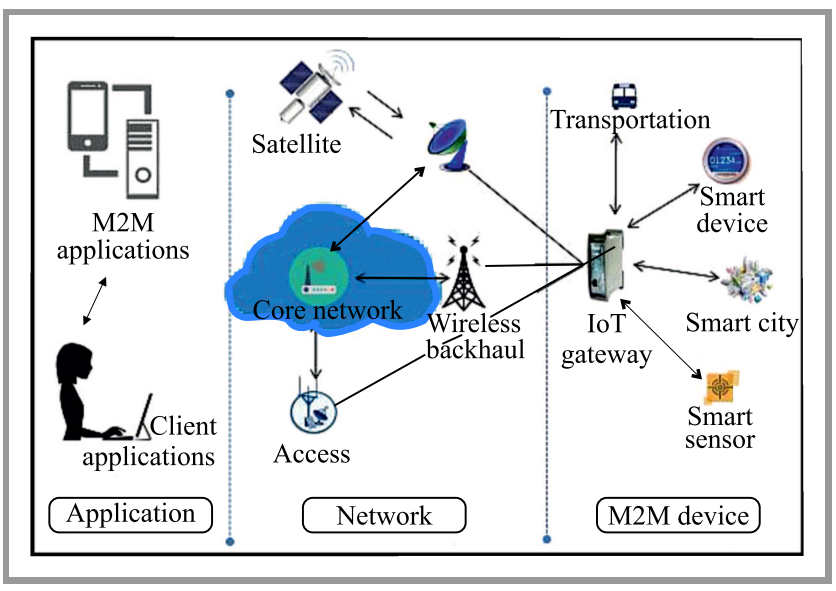

Fig. 1. Communications scheme in IoT.

and healthcare, public safety and environment monitoring, smart home/building, smart grid, smart mobility and smart tourism, industrial processing, agriculture, breeding, logistics, and product lifetime management.

- Core network. Specifies various access points (through wireless/wired) which transmits and receives data from gateways.

- Applications. Includes M2M and client applications. Applications are software components which acts as interface between an IoT system and users. These applications facilitate the users by giving options to monitor and control their devices (while they are connected to a network of similar devices, for example, homes or cars and controlled by a central system).

Therefore, the data sensed or gathered by various devices is accessed through wireless or wired network by gateways and connected to the end users of various applications where data is monitored and controlled.

Dynamic innovations in the fields from wireless sensors to nanotechnology are required for the development an IoT system. However, without comprehensive cognitive capability, IoT is just like an awkward stegosaurus, all brawn and no brains. To fulfill its potential and deal with growing 
Table 1

Comparative analysis of the performance parameters employed in IoT applications

\begin{tabular}{|l|c|c|c|c|c|c|c|}
\hline \multicolumn{1}{|c|}{ Author } & $\begin{array}{c}\text { Energy } \\
\text { consumption }\end{array}$ & Life time & $\begin{array}{c}\text { Resource } \\
\text { allocation }\end{array}$ & Throughput & Response time & $\begin{array}{c}\text { Quality } \\
\text { of Service }\end{array}$ & Delay \\
\hline \hline Li et al. [4] & Low & - & Good & - & - & - & High \\
\hline Hong et al. [5] & Low & - & - & - & Low & - & - \\
\hline Baloch et al. [6] & Low & - & Poor & - & High & Better & - \\
\hline Zhu et al. [7] & Low & - & - & High & - & Good & - \\
\hline Yang et al. [8] & Low & - & Good & - & - & - & - \\
\hline Bhardwaj et al. $[9]$ & Low & - & Good & High & - & Good & Low \\
\hline Chen et al. [10] & Low & Better & - & High & - & - & High \\
\hline Naser et al. [11] & Low & - & - & - & Low & - & - \\
\hline Feng et al. [12] & - & - & - & Low & - & Better & - \\
\hline
\end{tabular}

Table 2

Comparison of few platforms used to build various applications in IoT and CIoT

\begin{tabular}{|c|c|c|c|}
\hline Platforms & Pros & Cons & Applications \\
\hline NS2 Simulator & $\begin{array}{l}\text { - relatively cheaper and can be } \\
\text { used in testing complex sce- } \\
\text { narios } \\
\text { - simulates across various pro- } \\
\text { tocols and platforms }\end{array}$ & $\begin{array}{l}\text { - simulating real system } \\
\text { cannot be relied } \\
\text { - uncertainty in results } \\
\text { statistics }\end{array}$ & $\begin{array}{l}\text { - can be used to demonstrate } \\
\text { data traffic from sensors to } \\
\text { host }\end{array}$ \\
\hline Cooja Simulator & $\begin{array}{l}\text { - can be used to visualize the } \\
\text { status of each node } \\
\text { - parameters like transmission } \\
\text { range, radio interferences, } \\
\text { propagation delays, receiver } \\
\text { power can be obtained }\end{array}$ & $\begin{array}{l}\text { - comparatively slower } \\
\text { emulation at the hard- } \\
\text { ware level }\end{array}$ & $\begin{array}{l}\text { - security key exchange bet- } \\
\text { ween nodes can be demon- } \\
\text { strated } \\
\text { - helps evaluate interoperabil- } \\
\text { ity among network stacks, } \\
\text { energy consumption }\end{array}$ \\
\hline Matlab tool & $\begin{array}{l}\text { - remove noise and outliers } \\
\text { - perform statistical analysis } \\
\text { on channel data and explore } \\
\text { trends }\end{array}$ & - it is a paid software tool & $\begin{array}{l}\text { - works with ESP8266 chip } \\
\text { and Raspberry Pie }\end{array}$ \\
\hline $\begin{array}{l}\text { Opportunistic Network } \\
\text { Environment (ONE) } \\
\text { simulator }\end{array}$ & $\begin{array}{l}\text { - generates node motion using } \\
\text { movement models } \\
\text { - helps in visualizing both mo- } \\
\text { bility and message passing in } \\
\text { real time with a GUI }\end{array}$ & $\begin{array}{l}\text { - no physical and MCA } \\
\text { layer support } \\
\text { - event can happen only } \\
\text { before or after simula- } \\
\text { tion }\end{array}$ & $\begin{array}{l}\text { - used in demonstrating DTN } \\
\text { protocols to analyze delays } \\
\text { in data transfer of no end-to- } \\
\text { end paths communication }\end{array}$ \\
\hline Java programming & $\begin{array}{l}\text { - require less processing power } \\
\text { - portable across various hard- } \\
\text { ware platforms }\end{array}$ & $\begin{array}{l}\text { - compiler is not well } \\
\text { optimized } \\
\text { - byte-code interpretation } \\
\text { is slow }\end{array}$ & $\begin{array}{l}\text { - can be used to run embedded } \\
\text { and small devices }\end{array}$ \\
\hline $\mathrm{C}++$ & $\begin{array}{l}\text { - capable of handling complex } \\
\text { tasks with high speed of } \\
\text { execution } \\
\text { - hardware level programming } \\
\text { is comparatively simpler }\end{array}$ & $\begin{array}{l}\text { - no sophisticated garbage } \\
\text { collection } \\
\text { - strongly typed language } \\
\text { aids in remembering all } \\
\text { syntaxes }\end{array}$ & $\begin{array}{l}\text { - used in developing resource } \\
\text { constrained devices } \\
\text { - useful for embedded system } \\
\text { developers }\end{array}$ \\
\hline
\end{tabular}

challenges, we must take the cognitive capability into consideration and empower IoT with high-level intelligence, i.e., brain-empowered IoT or CIoT.

In this paper, we have analyzed some measures like energy consumption, network life time, resource allocation, throughput, time duration, quality of service and delay researched by various authors (Table 1). Since their vital role in any network, we tried to consider and compared these parameters, which would give us the scope to consider their importance while designing and implementing/simulating 
methodology for data perception in research work. Some of the parameters are considered for performance of IoT applications, these are shown in Table 1:

- Energy consumption. The energy is the major constraint resource of IoT/CIoT. The energy consumption is based on the amount of data perception or data processing or data gathering by the various nodes in the environment. Which will also depend on the applications used in the environment.

- Network lifetime. It is defined as the increase the probability of cognitive approaches in IoT as the increase the network lifetime of IoT. This is the major important metrics for the networks evaluation. The network lifetime parameter is also depending upon the efficient resources utilization in IoT/CIoT environment.

- Resource allocation. The mechanism for allocating resources (could be bandwidth, energy, nodes, etc.) allows to perform better network lifetime and increase the fault tolerance of IoT/CIoT.

- Throughput. It is the average rate of successful message delivery over a network (i.e. the number of data sent and received successfully over the network). It is an important representation of the performance and quality of a network connection.

- Response time. Response time is a measure of the total elapsed time between the start and completion of a task. All IoT/CIoT objects should be responding within the time constraints. There are two types of response time in IoT/CIoT like hard response time and soft response time. In hard response time, all IoT/CIoT objects should be responded to within the time constraints but should not violate the time constraints whereas soft response time, objects may violate the time constraints occasionally in the network environment.

- Quality of Service. QoS refers to any technology that manages data traffic to reduce packet loss, latency and jitter on the network. QoS controls and manages network resources by setting priorities for specific types of data.

- Network delay. The network delay specifies how long it takes for a bit of data to process across the network from one node to another.

In this paper, we have also identified few platforms used to build IoT applications, their pros and cons, and application (Table 2).

While the current development stage renders itself as a promising answer for enhancing availability, its reasonableness with regards to IoT is restricted for primary reasons are high cost, energy utilization, and lifetime. Currently, the most recognized attributes of IoT is that the expanding between network among general things or items and various intriguing services or applications. In any case, so far huge numbers of the current IoT applications are as yet subordinate exceedingly on individuals for cognition processing. This perception fills in as one of the essential inspirations of this paper to present CIoT, where general objects behave as agents and associate with physical environment as well as social communities with least human intercession.

\section{Cognitive IoT}

IoT is a technological revolution that brings into a new ubiquitous connectivity, computing, and communication era as described by Qihui et al. [13]. The fundamental concept is to equip any physical objects with sensors and collaborate with Internet to synchronize the physical and digital world, which is together to achieve many ubiquitous and pervasive computing-based applications and further used for the automation. The second generation of IoT is known as Cognitive Internet of Things (CIoT). Currently, the data gathered by the sensors/objects are not utilized properly interms of bandwidth, security, and privacy constraints as well as enormous unstructured data from logs and social frameworks are abundant in quantity of data. These data can be combined in a real time basis and valuable informations can be extracted by CIoT capabilities. The followings are the list of issues and challenges in CIoT mentioned by Dohr et al. [14]:

- standards for cross interoperability with heterogeneous networks,

- machine to machine standardization,

- self aware and self organizing networks,

- open framework for the CIoT,

- fault tolerance,

- adhoc deployable and configurable networks,

- dynamic and adaptable interoperability,

- open platform for CIoT,

- distributed energy efficient data processing,

- security and routing,

- access control and accounting schemes for IoT,

- low cost, secure, and high performance authentication,

- cognitive computing for IoT,

- context-aware computing,

- resource management for CIoT,

- connectivity, 
- power,

- adaptability,

- data perception,

- scalability.

In addition, the knowledge of IoT has been accentuated to an ever-increasing extent. After an intensive examination on the models of IoT, Zhang et al. [15] additionally trusted that the knowledge still can't fulfill the need of use. Subsequently, another idea of CIoT is proposed through coordinating insight into IoT. A CIoT is an IoT with cognitive capacity which is incorporated to advance execution and accomplish insight is characterized by Kasnesis et al. [16]. CIoT can apperceive current business writes and organize conditions, break down the apparent data in light of the earlier learning, make intelligent choices, and perform versatile and control activities, which aims to expand network execution and meet the application necessity.

In our investigation, we have incorporated cognition into IoT and present another idea of CIoT. The idea of CIoT is to allocate the intelligence in IoT. Cognition can impenetrate any parts of IoT and goes about as an essential part for a given application with a specific end goal to accomplish above examined points. The network model of CIoT is presented and it consists of three imperative parts showed up in Fig. 2:

- Access layer. Specifies the data access through internet from various sensors and actuators equipped in many physical devices and data from different

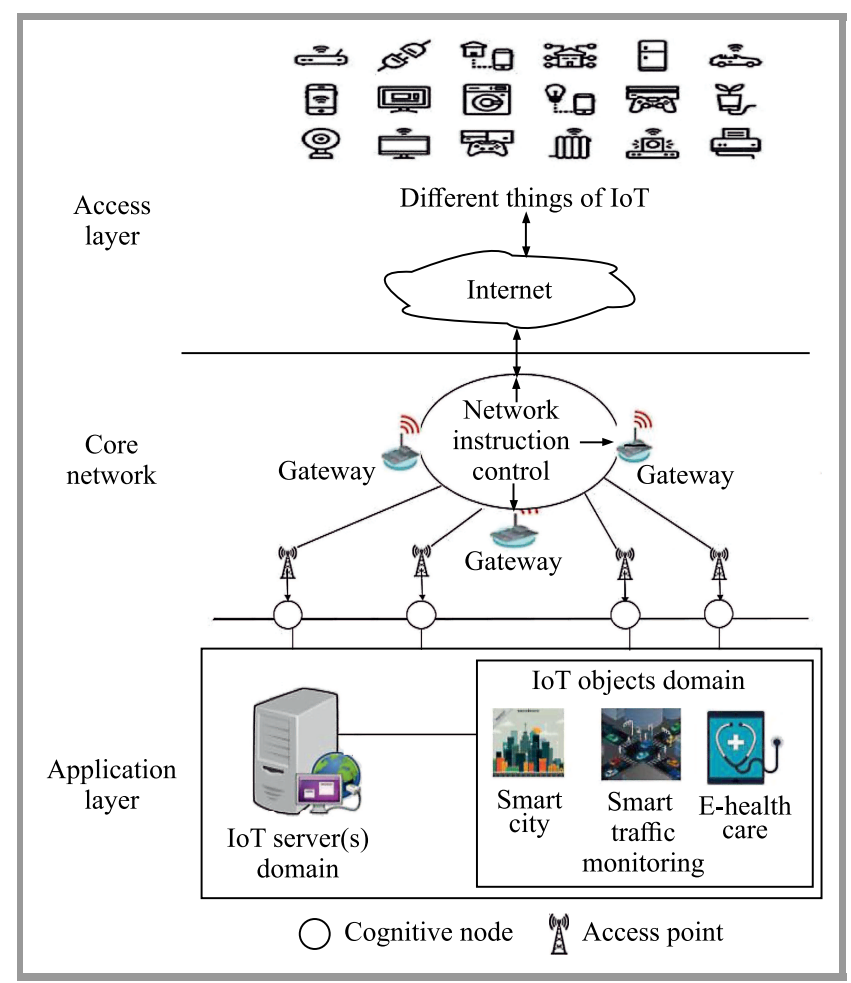

Fig. 2. CIoT network model. domains like medical and healthcare, databases. Data analysts make use of such data to find trends and gain actionable insights.

- Core network. Consists of network instruction control channel where in gateway that involves in preprocessing and filtering before moving it to the access points (to reduce the volume of data for detailed processing and storing) and transmits control commands going from the Internet to access points and finally to IoT based applications.

- Application layer. Cognitive agents having high reasoning capabilities process the data from core network and resolves real time problems that have a high degree of dynamism which is very essential for data perception in IoT. The solution to real time problems is realized in the various user applications like healthcare, traffic monitoring, and smart city/building.

The model incorporates core network and different access network spaces. The core network is principally comprised of access, remote, transmission routers, and so on. The access network spaces cognitive nodes, simple nodes, and different terminals. The implications of components are depicted in Fig. 2.

- CIoT objects domains. CIoT objects domains are high coupled and relatively independent. It is observed that, CIoT application consist at least one object domain but object domain can be divided into several sub-object domains. Based on the capabilities of data accessing nodes, these can be divided as cognitive nodes (CNs) and simple nodes (SNs). There are different numbers of CNs in different object domains and possible only one $\mathrm{CN}$ in an object domain under special circumstances. If object domain includes multiple $\mathrm{CNs}$, two or more $\mathrm{CNs}$ can work together to achieve goals according to requirements. Furthermore, two or more object domains can also cooperate if necessary.

- CIoT servers domain. Object domains can apperceive the crude data, however without the ability to manage it. The data will be sent to server running unique schedules to be handled. For the most part, servers for an application are coupled and faulttolerance, in this way, they are named as server domain. Now and then, server domain might be just a normal PC or a control terminal.

- Cognitive nodes. For multi domain cooperation (MDC), it alludes the particular $\mathrm{CN}$ to choose from every space to do agreeable assignments. There is diverse number of $\mathrm{CNs}$ in various spaces, possibly just a single. The demonstrating of CIoT design to locate new research thought for IoT and additionally an application which be acquainted with the proposed engineering and show that the work will have far expansive application prospect. 
Zhou et al. [17] has proposed a profound slant sentiment model inside the space of CIoT. Data recovery in social media is an overwhelming task which requires a great deal of specialized bits of knowledge. As a great instrument for large scale data recovery, hashing methods have likewise been widely utilized for content recovery. Deep sentiment hashing model is introduced, which is made out of following three stages are:

- a progressive consideration based long short-term memory network (LSTM) is prepared to acquire assessment particular report portrayals,

- given the record embeddings, $k$-nearest neighbor $(\mathrm{kNN})$ calculation is utilized to develop a Laplacian matrix which is anticipated into hash marks by means of Laplacian eigenmaps (LapEig) later,

- model for hash functions learning, which is regulated by both the produced hash names and the first sentiment names.

Such joint supervision guarantees that a definitive hash codes delivered by the learned hash functions keep up sentiment level closeness.

Zhan et al. [18] have proposed the cloud assisted CIoT in view of the registering and information storage capacities of cloud virtual machines, which make ensuring virtual machine, file system critical for the entire framework security. Agent less intermittent file system screens are ideal solutions for ensure cloud virtual machines on account of the safe and low-overhead highlights. Notwithstanding, the greater part of the intermittent screens generally check the majority of the virtual machine file system or ensured documents in each scanning survey, so plenty of secure records are examined over and over despite the fact that they are not defiled. A novel agent less occasional file system screen system is introduced for virtual machines with various image formats to enhance the execution of agent less intermittent screens. Cognitive radio with context of IoT is concerning a joined plan of future Internet and cognitive radio with respect to IoT. It proposes an embedded and simplicity agreeable spectrum identifying arrangement, which has been probably completed. Additionally, Alberti et al. [19] exhibited a dispersed programming-controlled sector aware spectrum distinguishing configuration to collect and dismember the range utilizes information. Finally, the proposed technique is joined to a future Internet configuration called NovaGenesis.

A device level multimodal data correlation mining (DMDC) demonstrate is quickly made in light out of the CCA to change the data incorporate into a subspace and dismember the data relationship. The relationship of the device is obtained in perspective of the intensive of data association and the region information of the device. By then a heterogeneous clustering model (HDC) is proposed by utilizing the result of the association examination to portray the device.
Lin et al. [20] proposed a device clustering algorithm in perspective of multimodal data correlation for CIoT, which joins the components of multimodal data association separate with device clustering. Somov et al. [21] proposed to improve crude sensor information with context data for facilitate utilization in CIoT. To do as such, authors have created virtual portrayals of real sensors called virtual items. These CIoT objects create crude sensor estimations, as well as enhance those with setting data also. The approach included, real sensor network organization for traffic checking in the city. Specifically, the work done exhibited how to improve crude information and how to separate knowledge from the accessible information.

The remainder of this paper is organized as follows. Section 3 presents the data perception in CIoT, pros and cons of the methods used for data perception by various authors. Section 4 presents architecture of cognitive agent and few data management techniques used by cognitive agent in CIoT. Section 5 describes proposed methodology for data perception in CIoT and applications.

\section{Data Perception in CIoT}

The current investigation in CIoT is far from the acknowledgment of conceptualized applications. There are different open research issues and difficulties are into this space and one of the significant issues in CIoT is data perception. Data perception is a procedure to guarantee the information being detected by the sensors that have better visuality as far as applications. It additionally guarantees that how well the information is recouped from the sensor network without having any redundancies. The accompanying is some one of a kind qualities of sensor information amid data perception like: heterogeneous, high dimension, contextaware information, and nonlinear reparability. Because of these blended qualities it is exceptionally testing to process enormous information in CIoT applications, which are coordinated various advances and protocols. The improvement of viable semantic advances and information revelation strategies is still in early stage. Thus, the information observation in CIoT is as yet an open issue.

The perception and access technique in perspective of IoT for hard assembling resources, computational assets, and insightful assets were analyzed by Tao et al. [22]. A fivelayered structure resource skillful perception and access framework in context IoT is arranged and shown. The key progressions for sharp perception and access of different assets (i.e. hard gathering and computational resources, and adroit resources) are depicted. Authors proposed another thought of CIoT and its relating insightful plan. CIoT contains protocol plane, cognitive plane and control plane modules and incorporates some novel arranged models, for instance, layer tradition stack, 3-layer cognitive rings. In paper [23], a number of STS sub-regions are found that respond selectively to certain types of social input, organized along a posterior to anterior axis. Authors also identified the regions of overlapping response to multiple contrasts, 
Table 3

The methods used for data perception by various authors

\begin{tabular}{|c|c|c|c|}
\hline Author & Method & Pros & Cons \\
\hline Benincas et al. [25] & $\begin{array}{l}\text { Cognitive-based percep- } \\
\text { tion oriented situation } \\
\text { awareness }\end{array}$ & $\begin{array}{l}\text { A novel approach, in view of the } \\
\text { idea of perception; situation aware- } \\
\text { ness improves basic decision-making } \\
\text { processes and gives capable results }\end{array}$ & $\begin{array}{l}\text { Enhancement of the proposed } \\
\text { approach for managing ex- } \\
\text { tremely versatile circumstances } \\
\text { can be done }\end{array}$ \\
\hline Cui et al. [26] & $\begin{array}{l}\text { Sparse representation in } \\
\text { cognitive based robotic } \\
\text { perception }\end{array}$ & $\begin{array}{l}\text { Intensively including learning algo- } \\
\text { rithms to accomplish highly adaptive } \\
\text { and intelligent behaviors, including } \\
\text { actuation, sensing, perception and } \\
\text { adaptive control }\end{array}$ & $\begin{array}{l}\text { Better execution is required as } \\
\text { far as perception }\end{array}$ \\
\hline Pappas et al. [27] & $\begin{array}{l}\text { Cognitive and affective } \\
\text { perceptions with fuzzy- } \\
\text { set qualitative compara- } \\
\text { tive analysis }\end{array}$ & $\begin{array}{l}\text { More interactive to target clients' } \\
\text { cognitive and affective perceptions, } \\
\text { and increment their expectation to } \\
\text { purchase }\end{array}$ & $\begin{array}{l}\text { Suggesting that distinctive sorts } \\
\text { of perceptions may co-exist so } \\
\text { as to enhance perception }\end{array}$ \\
\hline Gray et al. [28] & $\begin{array}{l}\text { A new approach in light } \\
\text { of fuzzy logic with cog- } \\
\text { nition; } \\
\text { Fuzzy cognitive mapping } \\
\text { (FCM) for stakeholder } \\
\text { perceptions }\end{array}$ & $\begin{array}{l}\text { Capable of evolving rapidly and } \\
\text { improved decision making } \\
\text { More accurate, less time consuming }\end{array}$ & $\begin{array}{l}\text { Improvements can be made to } \\
\text { refine and extend the utiliza- } \\
\text { tions of fuzzy logic method; } \\
\text { Limited life time }\end{array}$ \\
\hline Guirguis et al. [29] & $\begin{array}{l}\text { A multi-hop routing for } \\
\text { cognitive radio networks }\end{array}$ & $\begin{array}{l}\text { Better shows in good put routing } \\
\text { overhead, average size }\end{array}$ & $\begin{array}{l}\text { Leads to a higher aggregate } \\
\text { end-to-end delay }\end{array}$ \\
\hline Araujo et al. [30] & $\begin{array}{l}\text { Perception based assess- } \\
\text { ment for multidimen- } \\
\text { sional data visualizations }\end{array}$ & $\begin{array}{l}\text { Provides beginnings for further re- } \\
\text { search on the role of perception in } \\
\text { multidimensional data projections }\end{array}$ & $\begin{array}{l}\text { May expand this by investigat- } \\
\text { ing additional datasets, more } \\
\text { projection techniques }\end{array}$ \\
\hline Nitti et al. [31] & $\begin{array}{l}\text { SIoT (Social Internet of } \\
\text { Things) paradigm for the } \\
\text { sensing of channel status } \\
\text { to execute cognitive radio } \\
\text { solutions }\end{array}$ & $\begin{array}{l}\text { Enables faster and reliable informa- } \\
\text { tion/service }\end{array}$ & Less iterations are expected \\
\hline
\end{tabular}

including regions responsive to both language and theory of mind.

A creative approach presented by Chai et al. [24] relies upon fuzzy logic to show the fundamental cognitions and individual conduct standards of people on foot as deduced from perception in demand to evaluate age and sexual introduction effect of walkers in convergence a signalized crosswalk. Fuzzy sets and guidelines are made to show the association in the midst of human bits of knowledge and decisions of a man by walking. Through changing the enlistment components of different age and sexual introduction social events, individual direct principles of individuals by walking are surveyed and considered. Remarkable in connection to most past examinations, both more prepared and more energetic walkers are seen to be less risk taking than grown-up individuals by walking. Additionally, tremendous sexual orientation differentiates is found only for insights of most dangerous conditions.

As a summary the pros and cons of the methods used for data perception other authors are shown in Table 3.

\section{Cognitive Agents}

Cognitive agent (CA) is software that exhibits the core skills of a human brain to perform tasks like learning, reasoning, understanding, grasping and acquire knowledge. CAs involves in resolving real time problems that have a high degree of dynamism, which is, essential for data perception in CIoT as shown in Fig. 3. The following functions of CAs agents are:

- Beliefs. The beliefs represent to the data about the condition of the environment that is refreshed suitably after each detecting activity. The beliefs can be seen as the informative part of the framework state. Beliefs generate choices/options for desires.

- Desires. The desires are the motivational condition of the framework. They have data about the destinations to be expert, i.e. what needs or adjustments are related with the different current destinations. The way that the agent has a desire does not imply that the agent will do it. The agent completes 


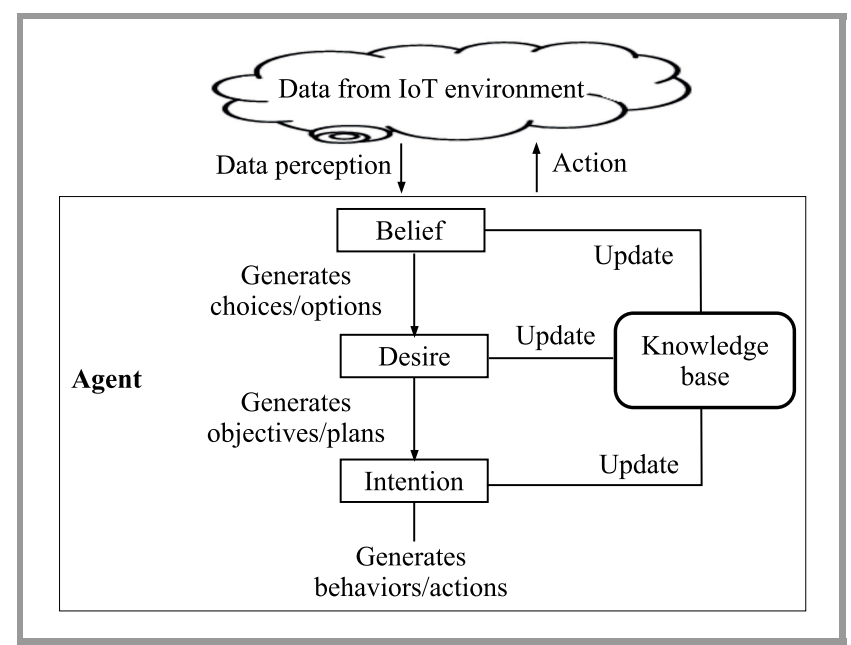

Fig. 3. Architecture of cognitive agents.

a deliberative procedure in which it stands up to its desires and beliefs and picks a set of desires that can be fulfilled. Desires generate objectives/plans to be implemented.

- Intention. The intention is a desire that is executed by a plan, since it can be done by the agents' beliefs (since it isn't rational that the agent completes something that it doesn't believe). Intentions generate actions as an output of this model architecture.

- Knowledge base. This is a repository that represents the agent's intentions on the basis of its current beliefs, desires, and intentions.

The desires can be conflicting to each other but not the intentions. The intentions represent the presently picked course of action. The intentions are insistent. An agent won't abandon its intentions - they will persist, until the point when the agent believes it has effectively accomplished them. In CIoT, the agent's techniques and behavior are depicted as the agent's beliefs. The choice of what to do and when to do it are the desires and intentions of the agent. Along these lines, a determined methodology (belief) of the agent is initiated if a desire of the agent turns into an intention. Followings are few data management techniques used by CAs in CIoT.

Bayesian networks. Perception is one of the required characteristics of cognitive process. The entire data that is perceived may not be important for cognitive process. Hence the classification of the perceived data is important and ideal, which is beneficial in saving working memory and ideal in long term memory. Thereby performance of the cognitive process can be enhanced as only relevant information is made available. The process of classification is carried out based on BDI model (belief, desire and intention) model using naive Bayes classifier is presented by Vishwanath et al. [32]. SWI-Prolog is used for the implementation purpose and results obtained, successfully clas- sified perceived data as efficient and trivial perception based on BDI model using naive Bayes by first estimating the prior probability for effective and trivial perception and then likelihood probability. Anchisi et al. [33] have proposed that placebo analgesia as an aspect of pain perception, others being the modulating effects of emotions, cognition and past experience. Here authors suggest that computational considerations of pain may present a unifying justification of these phenomena. It is also shown how Bayesian decision theory can explain for such features which are described by a model of pain that is successfully tested against experimental data.

Fuzzy logic. Data perception using fuzzy cognitive mapping (FCM) has been used as a part of a different course of action of contexts ranging from fisheries organization to agricultural development, with a final objective to create clear graphical models of complex structures presented by Gray et al. [34]. This extension in reputation is an after effect of FCM's base up approach and its ability to merge an extent of individual, group level and master learning into an open and organized course of action. Regardless of the way that there has been an extension in the usage of FCM as ecological planning and learning device, limited progress has been made with regards to the methodology's relationship to existing adaptability structures and how the use of FCM contrasts and other participatory showing/approaches open. Li et al. [35] have composed a transmission scheduling technique named cognitive access for regular topology (CART) in cognitive radio sensor systems (CRSNs) with insignificant control of channels and high transmission productivity. CART comprises of a timeslot and channel allotment method, an agreeable spectrum sensing plan, and a plan for detailing spectrum sensing results (SSRs). This paper likewise dissects CART's agreeable spectrum sensing for various transmission interference range and scope designs.

Neural networks. The multimedia neural cognitive computing (MNCC) model was designed by Liu et al. [36] based on the nervous mechanism and cognitive architecture. Furthermore, the semantic-oriented hierarchical cross-modal neural cognitive computing (CNCC) framework was proposed based on MNCC model and formal description and analysis for CNCC framework was given. It would effectively improve the performance of semantic processing for multimedia and cross-modal information. Zhang et al. [37] have proposed a unified deep learning based framework that enables effective human-thing cognitive interactivity in order to bridge individuals and IoT objects. They designed a reinforcement learning based selective attention mechanism (SAM) to discover the distinctive features from the input brain signals. In addition, authors proposed a modified long short-term memory (LSTM) to distinguish the inter-dimensional information forwarded from the SAM. Two practical real-time human-thing cognitive interaction applications were presented to validate the feasibility of their approach. 
Context aware computing. Jamnal et al. [38] have proposed a new form of ambient intelligent architecture for smart homes by anticipating occupant's intentions for understanding purpose. This is achieved with a combined knowledge from traditional context aware pervasive systems and present epoch of CIoT technologies. Architecture of ambient cognition model (ACM) is prepared with artificial intelligence machine learning algorithms of subtractive clustering and hidden Markov model to identify the patterns in daily activity living. The main objective of ACM model is to provide comfortable living environment for occupant. This model can be successfully adopted in elderly health care systems, smart classrooms, and smart spaces for automated environment.

Knowledge structure tree. Cognitive model-based evolutionary mechanism was presented by Lu et al. [39] with the knowledge structure tree (KST). The information present in IoT was analyzed by mimicking the information processing system of human brain and the evolutionary mechanism of information status based on cognitive model was explained. The analyzed information can play a vital role for the implementation of intelligent information processing under the environment of IoT.

Clustering. Zhang et al. [40] have presented spectrum sensing method based on clustering algorithm and signal characteristic is proposed for cognitive wireless multimedia sensor networks. Initially the wireless communication signal characteristics were acquired according to the sampling signal covariance matrix. Then, the clustering algorithm was used to classify and test the signal characteristics. The results from the experiment conducted were sat- isfactory and convinced about better sensing performance. Belk et al. [41] have focused on modeling users' cognitive approach on a set of web usage mining methods on navigation patterns and clickstream data. The purpose is to investigate whether k-means clustering can group users of particular cognitive approach by using measures acquired from a series of psychometric tests and content navigation activities. Three navigation metrics are proposed and used to find identifiable groups of users which have comparable navigation patterns in relation to their cognitive approach. The results of the experiment have been assessed with a user study which entailed a psychometric-based method for extracting the users' cognitive approach, combined with a real usage scenario of users navigating in a controlled web environment.

\section{Proposed Methodology}

The prime goal of the proposed study is to introduce a novel analytical modeling of data perception in CIoT for ensuring a robust, scalable, and cost-effective big data utilization. The paradigm of the proposed methodology is as follows (Fig. 4):

1. Deployment of wireless sensor network randomly.

2. Deployment of CAs in the WSNs.

3. Member nodes forward the context data to $\mathrm{CH}$ where $\mathrm{CA}$ is active.

4. $\mathrm{CH}$ after receiving data applies statistical measures to eliminate redundancies and forward the uniform data to gateway, which are further queued.

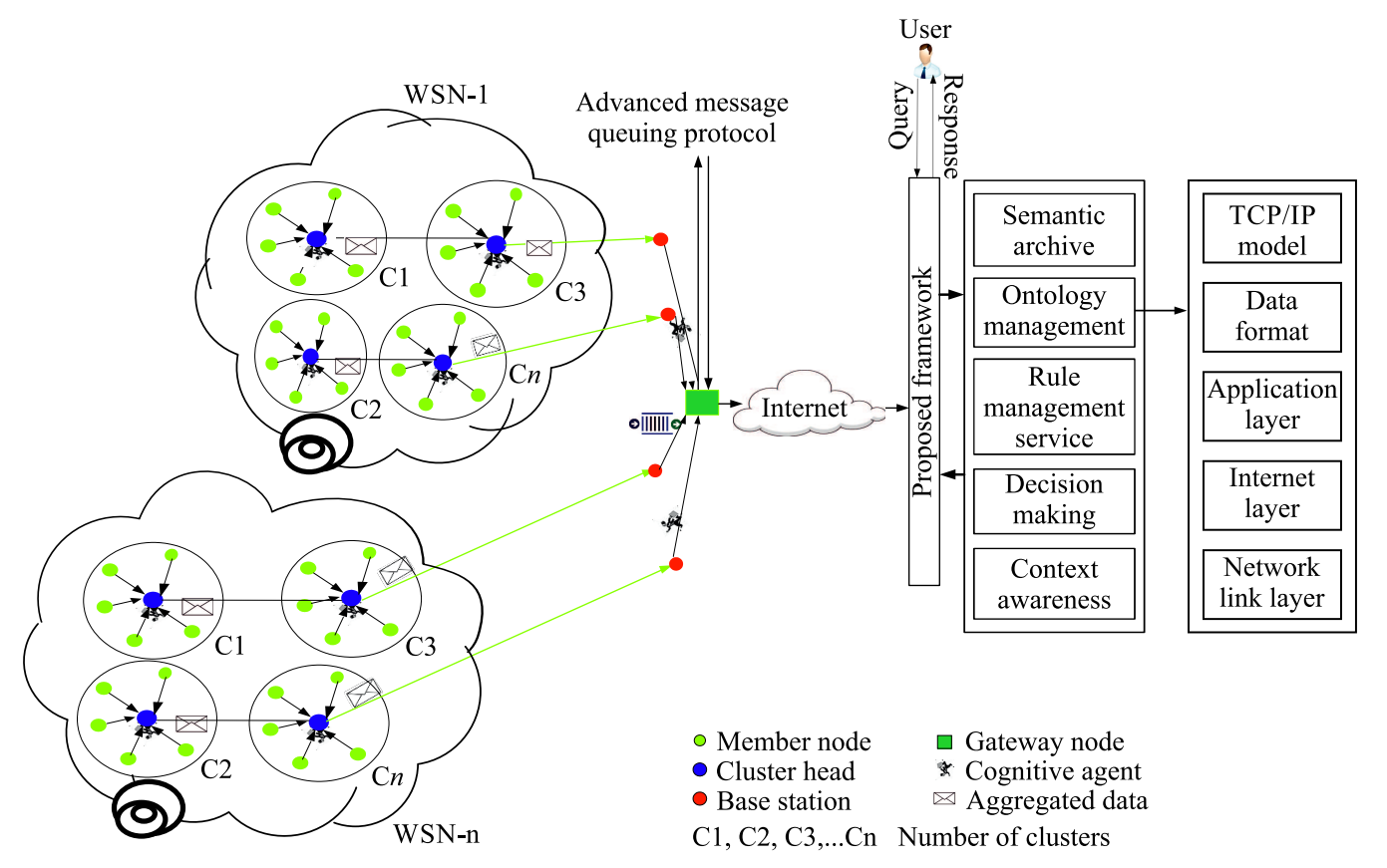

Fig. 4. Framework of data perception control using CA in CIoT. (For color pictures visit www.nit.eu/publications/journal-jtit) 
5. Gateway implements advanced message queuing protocol to process all the incoming queues of data from CA.

6. The filtered aggregated data is then forwarded to the proposed framework via gateway where ontological operations are carried out followed by semantic operation.

7. The cognitive agent performs the rule management services to construct and impose rules (businessbased rules) of data perception.

8. The processed data from rule management assists in decision making by using comparative analysis (incoming data and rule set) in CIoT.

9. Finally, the system model multiple contexts of data to further confirm the success factor of the rules (in order to avoid false positive).

10. Role of user is to query a particular request and receive specific and accurate response from the framework. The system is independent of any user-intervention.

Figure 5 illustrates the layout of proposed cognitive layered architectural model, the model consists of five layers: physical, data-perception, network, cognitive comput- ing, and application layer. The physical layer is associated with different heterogeneous (data sensed by various things/devices) WSNs. The access points are used as network administrators to access data sensed by physical layer. It's just not enough to collect the data from the access points but data should be addressed for various data characteristics like validity, completeness, accuracy, consistency, availability, and timeliness. Data perception layer consists of set of data functions like gathering, mapping, management, monitoring, merging, and semantic-ontology.

Cognitive nodes of network layer have cognitive computing features like:

- adaptive (ability to adjust to any surroundings),

- highly interactive (easy interaction with users),

- stateful (up-to-date),

- contextual (understand, identify the meaning).

Application layer includes different application domains like smart health care and monitoring, smart city/building, and so on. Finally, end user can access the CIoT applications. The objective of cognitive layer is to provide continuous services to users.

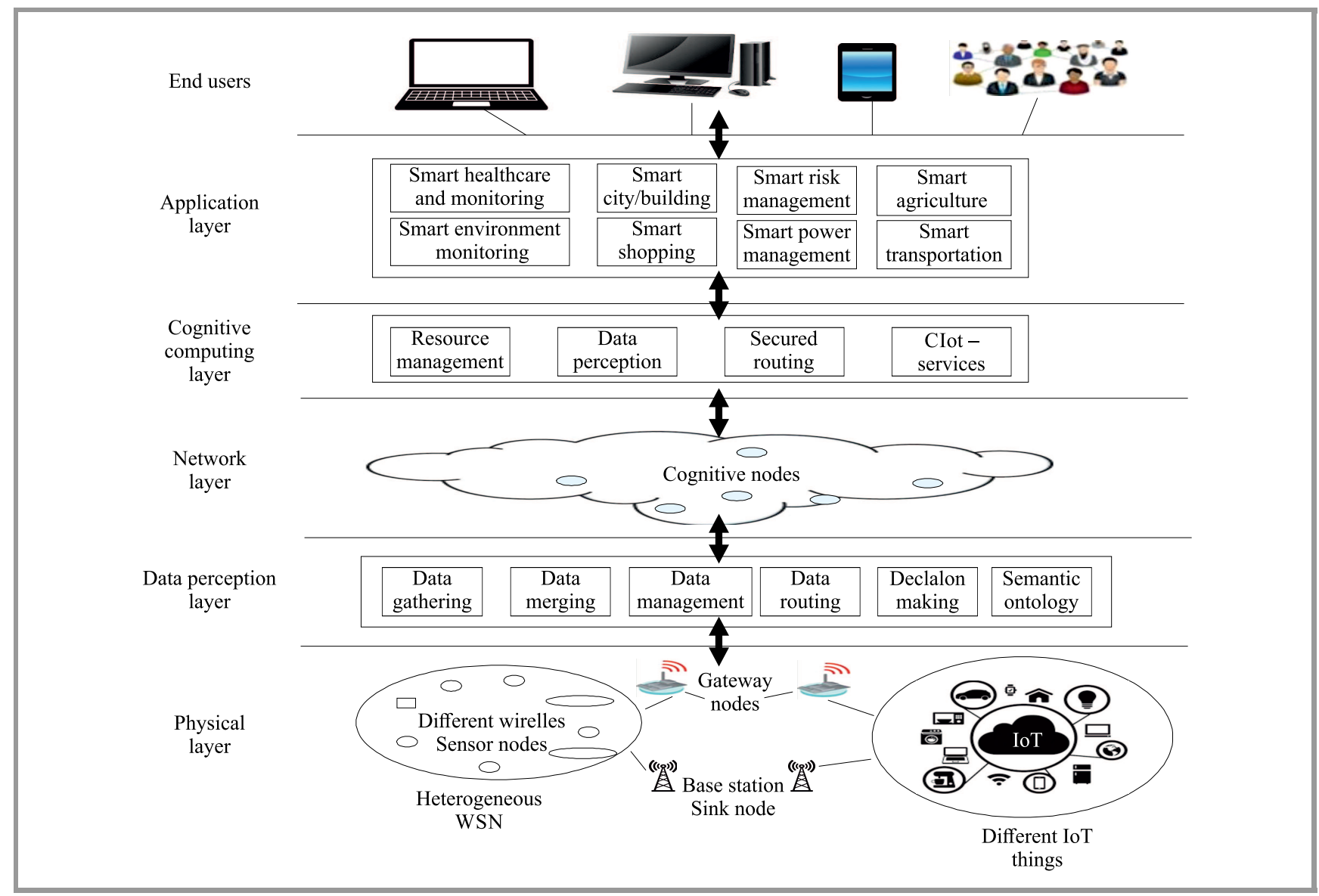

Fig. 5. Proposed layered architectural model in CIoT. 


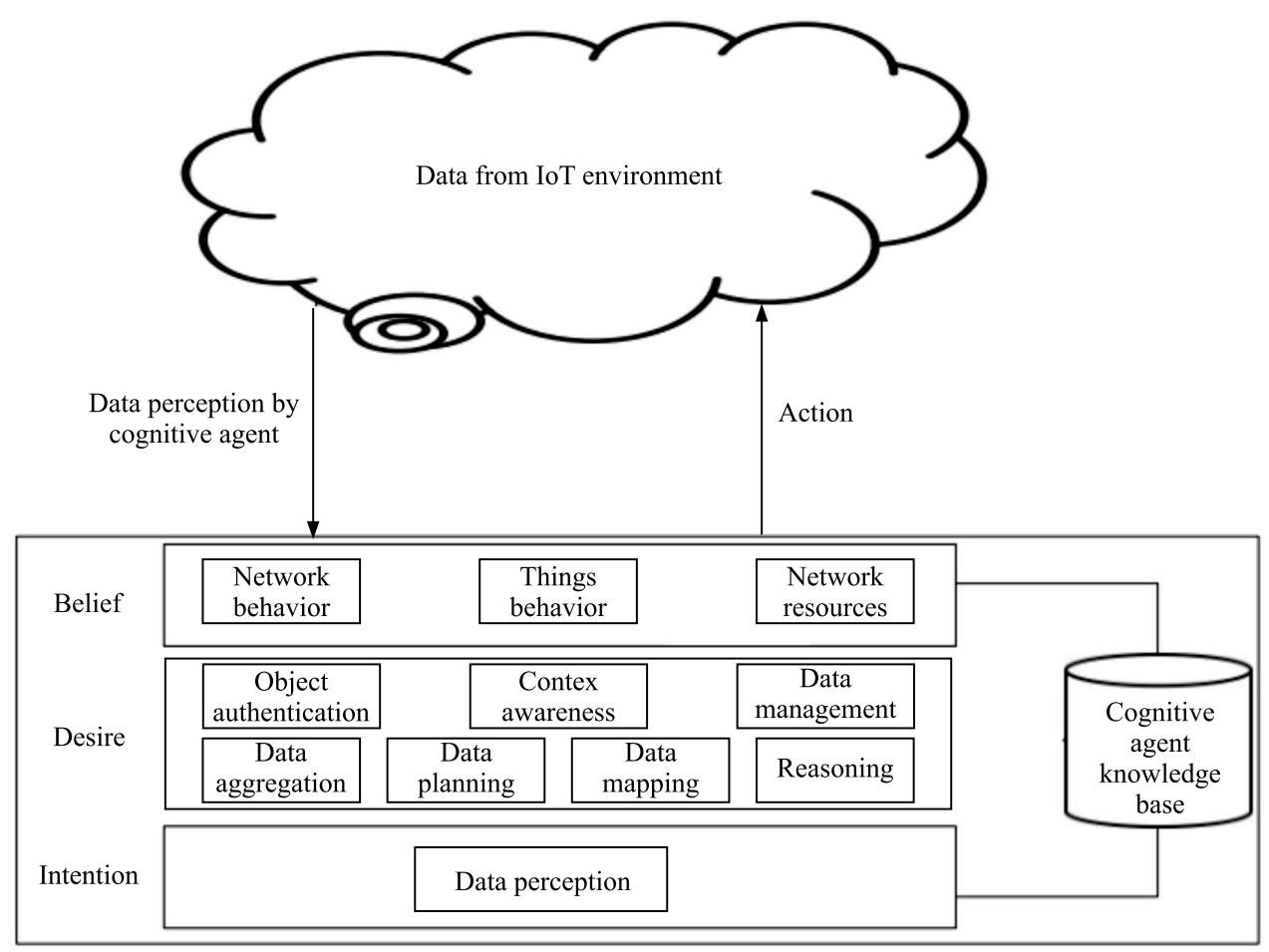

Fig. 6. Framework of cognitive agent BDI model for data perception.

\subsection{Proposed Cognitive Agent BDI Model for Data Perception}

The belief desire intention (BDI) model demonstration is given in Fig. 6. The cognitive agent constructs the belief model based on the data sensed or gathered by WSNs. CAs set the belief of the users based on the network behavior, things (from IoT) behavior and network resources. It is assumed that user is accessing the data from proper location and use the available network and devices. Based on the belief model, CA set the goals of the current environments, i.e. object authentication, context awareness, data management, data aggregation. Finally, agent defines the intentions of the environment which is to achieve data perception.

\section{Conclusion}

This paper describes the wide-ranging introduction of IoT and CIoT which covers issues and challenges and comparative analysis employed in IoT against the performance parameters like energy consumption, life time, resource allocation, throughput, time duration, quality of service, and delay. Also discussed CIoT environment reviewed on various pros and cons of different approaches for data perception in IoT environment. The review of cognitive agent based data perception is to present a new analytical demonstrating of data perception in CIoT for ensuring an effective use of big data. Presented the proposed methodology and model for data perception in CIoT using cognitive agents, provide benefits like minimizing and simplifying the data process and minimizing handling of sensor data, ensure better quality of experience, better quality of information and more.

\section{References}

[1] R. Minerva and A. B. Chebudie, "Towards a definition of the Internet of Things (IoT)", Technical Report, IEEE Internet Initiative, May 2015 [Online]. Available: https://iot.ieee.org/images/files/pdf/ IEEE_IoT_Towards_Definition_Internet_of_Things_Revision1_ 27MAY15.pdf

[2] J. Gubbi, R. Buyya, S. Marusic, and M. Palaniswami, "Internet of Things (IoT): A vision, architectural elements, and future directions", J. of Future Gener. Comp. Syst., vol. 29, no. 7, pp. 1645-1660, 2013 (doi: 10.1016/j.future.2013.01.010).

[3] N. C. Luong et al., "Data collection and wireless communication in Internet of Things (IoTs) using economic analysis and pricing models: a survey", IEEE Commun. Surv. \& Tutor., vol. 18, no. 4, pp. 2546-2590, 2016 (doi: 10.1109/COMST.2016.2582841).

[4] W. Li et. al., "Performance comparison of cognitive radio sensor networks for industrial IoT with different deployment patterns", IEEE Systems J., vol. 11, no. 3, pp. 1456-1466, 2017 (doi: 10.1109/JSYST.2015.1500518).

[5] G. J. Hong, S. Lee, J. Lim, W. Yoon, and S. Han, "RF spectrum sensing receiver system with improved frequency channel selectivity for Cognitive IoT Sensor Network applications", in Proc. of IEEE MTT-S Int. Microw. Symp. IMS 2016, San Francisco, CA, USA, 2016 (doi: 10.1109/MWSYM.2016.7540311).

[6] Z. Baloch, F. K. Shaikh, and M. A. Unar, "A context aware data fusion approach for health-IoT", Int. J. of Inform. Technol., vol. 10, no. 3, pp. 241-245, 2018 (doi:10.1007/s41870-018-0116-1).

[7] J. Zhu, Y. Song, D. Jiang, and H. Song, "Multi-armed bandit channel access scheme with cognitive radio technology in wireless sensor networks for the Internet of Things", J. on IEEE Access, vol. 4, pp. 4609-4617, 2016 (doi: 10.1109/ACCESS.2016.2600633). 
[8] W. Yang and X. Zhao, "Robust resource allocation for orthogonal frequency division multiplexing based cooperative cognitive radio networks with imperfect channel state information", J. of IET Commun., vol. 11, no. 2, pp. 273-281, 2016 (doi: 10.1049/iet-com.2016.0742).

[9] P. Bhardwaj, A. Panwar, and O. Ozdemir, "Enhanced dynamic spectrum access in multiband cognitive radio networks via optimized resource allocation", IEEE Trans. on Wirel. Commun., vol. 15, no. 12, pp. 8083-8106, 2016 (doi: 10.1109/TWC.2016.2612627).

[10] Z. Chen, H. Wang, Y. Liu, F. Bu, and Z. Wei, "A context aware routing protocol on Internet of Things based on sea computing model", J. of Computers, vol. 7, no. 1, pp. 96-105, 2012 (doi: $10.4304 /$ jcp.7.1.96-106)

[11] M. Z. Naser and V. K. R. Kodur, "Cognitive Infrastructure - a modern concept for resilient performance under extreme event", Autom. in Construction, vol. 90, pp. 253-264, 2018 (doi: 10.1016/j.autcon.2018.03.004)

[12] S. Feng, P. Setoodeh, and S. Haykin, "Smart home: cognitive interactive people-centric Internet of Things", IEEE Commun. Mag., vol. 55 , no. 2 , pp. 34-39, 2017 (doi: 10.1109/MCOM.2017.1600682CM)

[13] W. Qihui et al., "Cognitive Internet of Things: A new paradigm beyond connection", IEEE J. on Internet of Things, vol. 1, no. 2, pp. 129-143, 2014 (doi: 10.1109/JIOT.2014.2311513).

[14] A. Dohr, R. Modre-Opsrian, M. Drobics, D. Hayn, and G. Schreier, "The Internet of Things for ambient assisted living", in Proc. of 7th Int. Conf. on Inform. Technol.: New Generation, Las Vegas, NV, USA, 2010, pp. 804-809 (doi: 10.1109/ITNG.2010.104).

[15] M. Zhang et al., "A novel architecture for cognitive Internet of Things", Int. J. of Secur. and its Appl., vol. 9, no. 9, pp. 235-252, 2015 (doi: 10.1425/9ijsia.2015.9.9.21).

[16] P. Kasnesis, C. Z. Patrikakis, D. Kogias, L. Toumanidis, and I. S. Venieris, "Cognitive Friendship and Goal Management for the Social IoT", Computers and Elec. Engin., vol. 58, pp. 412-428, 2017 (doi: 10.1016/j.compeleceng.2016.09.024).

[17] K. Zhou, J. Zeng, Y. Liu, and F. Zou, "Deep sentiment hashing for text retrieval in social CIoT", J. of Future Gener. Comp. Syst., vol. 28, pp. 362-371, 2018 (doi: 10.1016/j.future.2018.03.047).

[18] D. Zhan et al., "A high-performance virtual machine file system monitor in cloud-assisted cognitive IoT", J. of Future Gener. Comp. Syst., vol. 88, pp. 209-219, 2018 (doi: 10.1016/j.future.2018.05.055)

[19] A. M. Alberti et al., "Cognitive radio in the context of Internet of Things using a novel future internet architecture called NovaGenesis", J. of Computers and Elec. Engin., vol. 57, pp. 147-161, 2017 (doi: 10.1016/j.comeleceng.2016.07.008).

[20] K. Lin, D. Wang, F. Xia, and H. Ge, "Device clustering algorithm based on multimodal data correlation in Cognitive Internet of Things", IEEE Internet of Things J., vol 5, no. 4, pp. 2263-2271, 2017 (doi: 10.1109/JIOT.2017.2728705).

[21] A. Somov, C. Dupont, and R. Giaffreda, "Supporting smart city mobility with Cognitive Internet of Things", in Proc. Future Network \& Mobile Summit, Lisboa, Portugal, 2013 (ISBN: 978-1-905824-37-3).

[22] F. Tao, Y. Zuo, L. D. Xu, and L. Zhang, "IoT-based intelligent perception and access of manufacturing resource toward cloud manufacturing", IEEE Trans. on Indust. Informat., vol. 10, no. 2, pp. 1547-1557, 2014 (doi: 10.1109/TII.2014.2306397).

[23] B. Deen, K. Koldewyn, N. Kanwisher, and R. Saxe, "Functional organization of social perception and cognition in the superior temporal sulcus", Cerebral Cortex, vol. 25, no. 11, pp. 4596-4609, 2015 (doi: 10.1093/cercor/bhv111).

[24] C. Chai, X. Shi, Y. D. Wong, M. J. Er, and E. T. Meng, "Fuzzy logicbased observation and evaluation of pedestrians behavioral patterns by age and gender", Transportat. Res. Part F: Traffic Psychol. and Behav., vol. 40, pp. 104-118, 2016 (doi: 10.1016/j.trf.2016.04.004).

[25] G. Benincasa, G. D’Aniello, C. De Maio, V. Loia, and F. Orciuoli, "Towards perception-oriented situation awareness systems", in Proc. of the 7th IEEE Int. Conf. Intelligent Syst. IS'2014, Warsaw, Poland, 2014, pp. 813-824 (doi: 10.007/978-3-319-11313-5_71).
[26] Z. Cui, S. S. Ge, Z. Cao, J. Yang, and H. Ren, "Analysis of different sparsity methods in constrained RBM for sparse representation in cognitive robotic perception", J. of Intell. and Robotic Syst., vol. 80, no. 1, pp. 121-132, 2015 (doi: 10.1007/s10846-015-0213-3).

[27] I. O. Pappas, P. E. Kourouthanassis, M. N. Giannakos, and V. Chrissikopoulos, "Explaining online shopping behavior with fsQCA: the role of cognitive and affective perceptions", J. of Business Res., vol. 69 , no. 2, pp. 794-803, 2016 (doi: 10.1016/j.jbusres.2015.07.010)

[28] S. R. Gray et al., "Are coastal managers detecting the problem? Assessing stakeholder perception of climate vulnerability using fuzzy cognitive mapping", Ocean \& Coastal Manag., vol. 94, pp. 74-89, 2014 (doi: 10.1016/j.ocecoaman.2013.11.008).

[29] A. Guirguis, M. Karmoose, K. Habak, M. El-Nainay, and M. Youssef, "Cooperation-based multi-hop routing protocol for cognitive radio networks", J. of Netw. and Comp. Appl., vol. 110, pp. 27-42, 2018 (doi: 10.1016/j.jnca.2018.03.005).

[30] H. D. S. Araújo et al., "A proposal for iot dynamic routes selection based on contextual information", Sensors, vol. 18, no. 2 , pp. 353-344, 2018 (doi: 10.3390/s18020353).

[31] M. Nitti, M. Murroni, M. Fadda, and L. Atzori, "Exploiting social Internet of Things features in cognitive radio", IEEE Access, vol. 4, pp. 9204-9212, 2016 (doi: 10.1109/ACCESS.2016.2645979).

[32] Y. Vishwanath, T. S. Murali, and M. V. Vijayakumar, "Implementation of perception classification based on BDI model using Bayesian classifier", in Proc. of Int. J. of Comp. Sci. and Inform. Technol. (IJCSIT), vol. 5, no. 6, pp. 8161-8165, 2014 [Online]. Available: http://ijcsit.com/docs/Volume\%205/vol5issue06/ ijcsit20140506282.pdf

[33] D. Anchisi and M. Zanon, "A Bayesian perspective on sensory and cognitive integration in pain perception and placebo analgesia", PLoS One, vol. 10, no. 2, pp. 1-12, 2015 (doi: 10.1371/journal.pone.0117270).

[34] S. A. Gray et al., "Using fuzzy cognitive mapping as a participatory approach to analyze change, preferred states, and perceived resilience of social-ecological systems", Ecology and Society, vol. 20, no. 2, pp. 1-10, 2015 (doi: 10.5751/ES-07396-200211).

[35] L. Wenxiang et al., "Performance comparison of cognitive radio sensor networks for industrial IoT with different deployment patterns", IEEE Systems J., vol. 11, no. 3, pp. 1456-1466, 2017 (doi: 10.1109/JSYST.2015.2500518).

[36] Y. Liu, "Research on the Brain-inspired Cross-media Neural Cognitive Computing Framework", 2018, arXiv:1805.01385 [Online]. Available: https://arxiv.org/ftp/arxiv/papers/1805/1805.01385.pdf

[37] X. Zhang, L. Yao, S. Zhang, S. S., Kanhere, Q. Z., Sheng, Y. Liu, "Internet of Things meets brain-computer interface: a unified deep learning framework for enabling human-thing cognitive interactivity", J. of IEEE Internet of Things, vol. 6, no. 2, pp. 2084-2092, 2018 (doi: 10.1109/JIOT.2018.2877786).

[38] G. Jamnal and X. D. Liu, "A cognitive-IoE approach to ambient intelligent smart home", in Proc. of the 2nd Int. Conf. on Internet of Things, Big Data and Secur. IoTBDS 2017, Porto, Portugal, 2017, vol. 2, pp. 302-308 (doi: 10.5220/0006304103020308).

[39] L. Huimin, L. Hu, G. Liu, and J. Zhou, "Cognitive Model-based evolution mechanism of the information status in Internet of Things", Inform. Technol. J., vol. 13, no. 15, pp. 2431-2436, 2014 (doi: 10.3923/itj.2014.2431.2436).

[40] Y. Zhang, P. W. S. Zhang, Y. Wang, and N. Li, "A spectrum sensing method based on signal feature and clustering algorithm in cognitive wireless multimedia sensor networks", Adv. in Multimed., vol. 2017, pp. 1-10, 2017 (doi: 10.1155/2017/2895680).

[41] M. Belk, E. Papatheocharous, P. Germanakos, and G. Samaras, "Investigating the relation between users cognitive style and web navigation behavior with K-means clustering", in Advances in Conceptual Modeling ER 2012 Workshops CMS, ECDM-NoCoDA, MoDIC, MORE-BI, RIGiM, SeCoGIS, WISM, Florence, Italy, October 15-18, 2012. Proceedings, S. Castano, P. Vassiliadis, L V. Lakshmanan, and M. L. Lee, Eds. LNCS, vol. 7518. Springer, 2012, pp. 337-346 (doi: 10.1007/978-3-642-33999-8_40). 


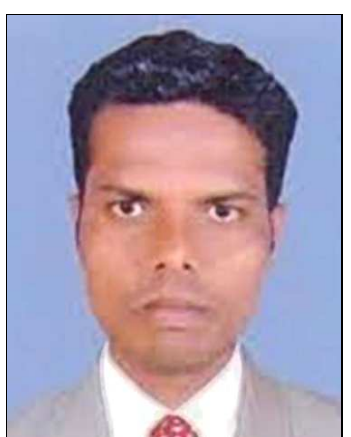

Lokesh B. Bhajantri received $\mathrm{Ph} . \mathrm{D}$. degree in Computer Science and Engineering from the Visveswaraiah Technological University (VTU), Belgaum, Karnataka, 2015. He is presently working as an Associate Professor in the Department of Information Science and Engineering, Basaveshwar Engineering College, Bagalkot, India, His areas of interests include distributed sensor networks, cognitive Internet of Things, mobile computing and communications, networking protocols, genetic algorithms, applications of agents and real time systems. He is a member of Board of Studies in the Department of Information Science and Engineering, Basaveshwar Engineering College, Bagalkot, Karnataka, India. He is a member of International Association of Computer Science and Information Technology (IACSIT).

E-mail: lokeshcse@yahoo.co.in

Department of Information Science and Engineering

Basaveshwar Engineering College

Bagalkot, Karnataka, India

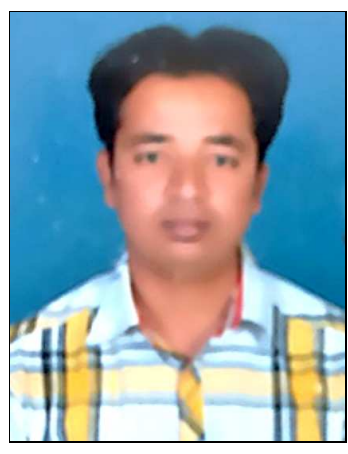

Prashant M. Baluragi received his M.Tech. degree in Computer Science and Engineering from the Basaveshwar Engineering College, Bagalkot under Visvesvaraya Technological University Belagavi, India. $\mathrm{He}$ is presently, working as an Assistant Professor in Department of Master of Computer Applications, KLE Institute of Technology, Hubballi, Karnataka, India. He has an experience of 5 years of teaching and an experience of 8 years in software industries (IBM India and TCS India). He is pursuing Ph.D. in the area of Cognitive Internet of Things (CIoT).

E-mail: prashant.baluragi@gmail.com

Department of Master of Computer Application

KLE Institute of Technology

Hubli, Karnataka, India 